\title{
Identificação de perfis em ferramentas de participação eletrônica: um estudo da ferramenta SoPa
}

\author{
Flávio B. S. Mota $^{1}$, Melise M. V. Paula ${ }^{1}$, Isabela N. Drummond ${ }^{2}$ \\ ${ }^{1}$ Programa de Pós-Graduação em Ciência e Tecnologia da Computação \\ Universidade Federal de Itajubá (UNIFEI) \\ Itajubá - MG - Brasil \\ ${ }^{2}$ Instituto de Matemática e Computação \\ Universidade Federal de Itajubá (UNIFEI) \\ Itajubá - MG - Brasil \\ \{flaviomota, melise, isadrummond\}@unifei.edu.br
}

\begin{abstract}
Electronic participation (e-participation) tools have emerged as initiatives to allow society to participate in decision-making in public administration. In this context, ways to increase citizen engagement become a problem to be investigated, since it is necessary to have an active participation by citizens. One way is to understand the profile of users of e-participation tools to identify different levels of engagement. This work aims to identify such profiles combining CRM (Customer Relationship Management) strategies and machine learning.
\end{abstract}

Resumo. Ferramentas de participação eletrônica (e-participação) têm surgido como iniciativas para permitir que a sociedade participe da tomada de decisão na administração pública. Neste contexto, formas de aumentar engajamento cidadão se torna uma problemática a ser investigada, visto que é necessário uma participação ativa por parte dos cidadãos. Uma das formas é o entendimento do perfil dos usuários de ferramentas de e-participação para identificar os diferentes niveis de engajamento. Este trabalho se propõe a identificar tais perfis combinando estrátegias de CRM (Customer Relationship Management) $e$ aprendizado de máquina.

\section{Introdução}

A difusão das Tecnologias de Informação e Comunicação (TICs) possibilitou o desenvolvimento de iniciativas ligadas a atividades sociais e políticas, entre elas a participação eletrônica (e-participação). A e-participação pode ser entendida como um meio que visa, através do uso das TICs, engajar os cidadãos na política e na tomada de decisão a fim de tornar a administração pública participativa, inclusiva, colaborativa e deliberativa [ONU 2018].

Apesar da variedade de ferramentas de e-particiação [Fernandes et al. 2018] e dos esforços governamentais na área das TICs, de acordo com o ranking definido pela UNPAN (United Nations Public Administration Network), o Brasil não está entre os principais países em nenhum nível de participação, o que demonstra a necessidade de investigar formas de aumentar o engajamento cidadão nessas ferramentas [Maciel et al. 2017]. 


\section{Apresentação do Problema}

De acordo com [Pade-Khene et al. 2017] e [Sibande e Thinyane 2016] o engajamento cidadão é uma problemática muito discutida em estudos sobre a e-participação. O engajamento cidadão pode ser entendido como a participação ativa dos cidadãos nos processos de tomada de decisão em parceria com o governo [OECD 2001]. Há muito interesse em ferramentas desse tipo por parte dos cidadãos, porém existem poucas ações práticas que conduzam a uma participação efetiva.

Segundo [No et al. 2016], apesar da pouca motivação dos cidadãos, a importância das ferramentas de e-participação reside no fato de que elas expandem o acesso e diminuem barreiras sociais, ao criar ambientes virtuais nos quais pessoas de diferentes extratos sociais podem opinar e participar.

[Reiersølmoen et al. 2018] argumentam que engajar uma grande parte do público no processo de tomada de decisão torna a participação mais efetiva e plural. Dessa forma, a busca por meios tecnológicos que estimulem a motivação e o engajamento deve ser multidisciplinar.

O trabalho de [Kopackova e Komarkova 2019] aborda a questão do engajamento cidadão em ferramentas onde é possível informar sobre problemas de infraesrutura pública. Os autores argumentam que o sucesso dessas ferramentas é altamente dependente da aceitação do usuário, algo que está relacionado com a usabilidade.

Já o trabalho de [Aristeidou et al. 2017] investiga a relação entre o engajamento e os padrões de comportamento em ferramentas que exigem maior participação cidadã. Através de uma análise de dados de logs e questionários, os autores definiram perfis e níveis de engajamento entre os usuários, permitindo elaborar diferentes abordagens para tais perfis.

\section{Proposta de Solução}

O objetivo da pesquisa é encontrar perfis de usuários em uma ferramenta de participação eletrônica que indiquem diferentes níveis de engajamento. Para tanto serão utilizadas estratégias de CRM (Customer Relationship Management) para a captura de dados da interação do usuário com a ferramenta, e modelos de aprendizado de máquina para análise de dados e identificação dos perfis. Além disso, será analisada a possibilidade de associar os padrões de comportamento identificados na ferramenta com perfis identificados por meio de teorias comportamentais como, por exemplo, a classificação tipológica de MyersBriggs [Myers e Myers 2010].

A ferramenta utilizada como estudo de caso da pesquisa será o sistema Sociedade Participativa (SoPa), cujo objetivo é expandir as possibilidades para a participação cidadã a partir de um ambiente virtual que facilite a discussão e reutilização do conhecimento criado [Caetano 2018]. Este artefato foi desenvolvido utilizando como base tecnológica as redes sociais.

A captura dos dados será realizada através de uma ferramenta de CRM que registra toda a interação do usuário com a interface do sistema, permitindo capturar eventos de clique e rolagem de página, tempo de permanência, páginas mais acessadas, fluxos de navegação etc. Ferramentas como essa são utilizadas por empresas para apoiar a descoberta de padrões de comportamento dos clientes e, posteriormente, permitir a 
recomendação de produtos e serviços [Cnudde e Martens 2015]. Além desses dados, um questionário será aplicado para coletar outras informações a respeito do comportamento dos usuários.

O processamento dos dados coletados será realizado através de algoritmos de aprendizado de máquina, visando gerar agrupamentos que possam identificar perfis de usuários com diferentes níveis de engajamento.Empregando os dados obtidos através do questionário, pretende-se utilizar um algoritmo de aprendizado que seja capaz de associar os perfis identificados àqueles presentes na teoria comportamental de Myers-Briggs.

Por fim, espera-se que o levantamento dos perfis possa servir como forma de identificar os diferentes níveis de engajamento cidadão na ferramenta, permitindo que diferentes abordagens sejam utilizadas para aumentar o grau de engajamento de determinados perfis. O trabalho pode ser caracterizado como um estudo de caso por abordar o processo de planejamento, coleta e análise de dados [Ventura 2007].

\section{Avaliação da Solução}

Através da aplicação de métricas de validação dos resultados obtidos no processo de identificação dos perfis, buscando investigar o quão representativos são os grupos encontrados nos conjuntos de dados. A avaliação desses resultados geralmente é realizada com índices estatísticos que validam de maneira qualitativa o mérito das estruturas encontradas [Faceli et al. 2005].

\section{Atividades Realizadas}

Foi realizado o levantamento de trabalhos relacionados com o objetivo dessa pesquisa nas bases de dados Scopus, Web of Science e IEEE. A partir dos trabalhos encontrados, um mapeamento sistemático está sendo elaborado.javascript

A ferramenta de captura de dados foi adicionada ao sistema SoPa, que será utilizado em parceria com o Núcleo Estratégico Interdisciplinar em Resiliência Urbana (Neiru), um projeto de extensão da Universidade Federal de Itajubá, para auxiliar no processo de elaboração de um Plano Municipal de Meio Ambiente da cidade de Pouso Alegre. Estratégias de divulgação do SoPa também foram definidas para fomentar a utilização por parte dos cidadãos, visando a obtenção de maior volume de dados.

\section{Conclusão}

Utilizando o processo de coleta automática de dados e algoritmos de aprendizado de máquina, fica proposta uma forma de identificar perfis de engajamento de usuários em ferramentas de participação eletrônica baseado em padrões de comportamento.

O trabalho contribui para as áreas de participação eletrônica e inteligência artificial, visto que se propõe a identificar perfis que podem ser utilizados para promover o engajamento cidadão em iniciativas de e-participação, através do uso de algoritmos de aprendizado de máquina que podem se mostrar mais ou menos adequados ao contexto estudado. 


\section{Referências}

Aristeidou, M., Scanlon, E., e Sharples, M. (2017). Profiles of engagement in online communities of citizen science participation. Computers in Human Behavior, 74:246256.

Caetano, B. P. (2018). Uma Proposta para Participação Cidadã na Solução de Problemas da Sociedade. Dissertação de Mestrado, Universidade Federal do Rio de Janeiro, Rio de Janeiro.

Cnudde, S. D. e Martens, D. (2015). Loyal to your city? A data mining analysis of a public service loyalty program. Decision Support Systems, 73:74-84.

Faceli, K., Carvalho, A. C. P. L. F. d., e Souto, M. C. P. d. (2005). Validação de algoritmos de agrupamento. Number 254, page 38.

Fernandes, L. S., Mota, F. B. S., Caetano, B. P., Silva, G. G., Paula, M. M. V. d., e Souza, J. M. D. (2018). Citizen participation: a diagnostic from the perspective of available tools. Iberian Conference on Information Systems and Technologies (CISTI), pages $1-6$.

Kopackova, H. e Komarkova, J. (2019). Participatory technologies in smart cities: What citizens want and how to ask them. Telematics and Informatics, 47.

Maciel, C., Cappelli, C., e Slaviero, C. (2017). Methodologies and Technologies for Citizen Participation. In I GranDSI-BR -Grand Research Challenges in Information Systems in Brazil 2016-2026, Special Committee on Information Systems (CE-SI), page 184. Brazilian Computer Society (SBC).

Myers, I. B. e Myers, P. B. (2010). Gifts Differing: Understanding Personality Type. Davies-Black, 2 edition.

No, W., Mook, L., e Schugurensky, D. (2016). Examining ideation processes in online invited spaces. In ACM International Conference Proceeding Series, volume 08-10June-2016, pages 67-75.

OECD (2001). Citizens as Partners: Information, Consultation and Public Participation in Policy-Making.

ONU (2018). E-government for the future we want. In United Nations e-government survey, pages 81-94.

Pade-Khene, C., Thinyane, H., e Machiri, M. (2017). Building foundations before technology: An operation model for digital citizen engagement in resource constrained contexts. In Proceedings of the European Conference on e-Government, ECEG, pages $118-126$.

Reiersølmoen, M., Gianni, F., e Divitini, M. (2018). DELTA: Promoting young people participation in urban planning. Smart Innovation, Systems and Technologies, 80:7789.

Sibande, R. e Thinyane, H. (2016). A mobile based technology platform for citizen engagement in Malawi. In 2016 IST-Africa Conference, IST-Africa 2016.

Ventura, M. M. (2007). O Estudo de Caso como Modalidade de Pesquisa. Rev SOCERJ, 20(5):383-386. 